\title{
Dropped head presentation in myotonic dystrophy type 1
}

Sir,

Dropped head syndrome, alternatively known as head ptosis or head drop, is caused by a number of neuromuscular conditions. ${ }^{[1,2]}$ The most common of these disorders include motor neuron disease, myasthenia gravis, and isolated neck extensor myopathy. This is the first report of a patient with genetically confirmed myotonic dystrophy type 1 (DM1) who presented with the dropped head syndrome.

A 61-year-old man presented with a 10-year history of slowly progressing neck extensor weakness. There was no family history of any neuromuscular disorder or other non-neurological features suggestive of DM1. Neurological examination revealed marked neck extensor weakness of Grade 1/5 (MRC scale). He also had mild weakness, Grade 4/5, of neck flexors and of the proximal muscles of arms and legs. Percussion myotonia 
was elicited in his abductor pollicis brevis muscles bilaterally. There was mild ptosis but the rest of the neurological examination was normal. Deep tendon reflexes and planters were normal. Sensory and mental status and cerebellar testing were normal. Neurophysiological testing disclosed normal motor and sensory nerve conduction velocities and needle electromyography revealed myotonic discharges in all muscles sampled. A clinical diagnosis of myotonic dystrophy (DM) was made and genetic testing was performed for both types 1 and 2 . The results of the test for DM2 were negative. Genetic testing for DM1 showed a genotype of 505 and 14 CGG trinucleotide repeats in alleles 1 and 2 (normal range: 5-37 CGG repeats) confirming the diagnosis.

Myopathies that have been reported to cause dropped head syndrome include both genetic diseases such as Becker's muscular dystrophy, nemaline rod and mitochondrial myopathy, and acquired disorders. ${ }^{[1,2]}$

Although neck flexor weakness is a common feature in both DM1 and DM2, predominant or severe neck extensor weakness is rare. ${ }^{[3]}$ There has been one report of dropped head syndrome developing in a patient with a clinical diagnosis of PROMM (proximal myotonic myopathy) or DM2. ${ }^{[4]}$ In that patient, genetic testing for DM1 was negative. However, since the genetic test for DM2 was not available at the time of the report, the clinical diagnosis remains genetically unconfirmed.

There have been no reports of patients with DM1 presenting with head drop. The reason for such localized presentation of a disorder that is electrophysiologically generalized in the muscles is not clear. It is possible that the variability in the degree of muscle weakness could be related to somatic heterogeneity of expansion mutation size. It may be that in our patient, there may be more muscle fibers containing expanded DM1 alleles relative to other muscles. Regardless of exact pathophysiology, this case study expands the spectrum of those neuromuscular disorders that can present with dropped head syndrome and the range of clinical manifestations of DM1.

\section{R. P. Grewal}

New Jersey Neuroscience Institute, JFK Medical Center, Edison, New Jersey, USA. E-mail: rgrewal@solarisha.org

\section{References}

1. Lomen-Hearth C, Simmons ML, DeArmond S, Layzer R. Adult-onset nemaline myopathy: Another cause of dropped head. Muscle Nerve 1999;22:1146-50.

2. Rahim R, Gupta D, Bertorini TE, LeDoux MS. Dropped Head Presentation of mitochondrial myopathy. J Clin Neuromuse Dis 2003;5:108-14.

3. Ranum LP, Day JW. Myotonic dystrophy: Clinical and molecular parallels between myotonic dystrophy type 1 and type 2. Curr Neurol Neurosci Rep $2002 ; 2: 465-70$

4. Evidente VGH, Cook A. Floppy head syndrome resulting from proximal myotonic muscular dystrophy. Ann Neurol 1997;42:17.

Accepted on 05-11-2004 